\title{
Specifying the antecedents of breast-feeding duration in Peru through a structural equation model
}

\author{
Rafael Pérez-Escamilla ${ }^{1, *, \dagger}$, José A Cobas ${ }^{2, \dagger}$, Hector Balcazar $^{3}$ and Mary Holland Benin ${ }^{2}$ \\ 'Department of Nutritional Sciences, University of Connecticut, Storrs, CT 06269-4017, USA: \\ ${ }^{2}$ Department of Sociology, Arizona State University, USA: ${ }^{3}$ Department of Family Resources and Human \\ Development, Arizona State University, USA
}

Submitted 23 November 1998: Accepted 4 June 1999

\begin{abstract}
Objective: To examine the effects of socioeconomic status and biocultural variables (planned pregnancy, prenatal care, timing of initiation of breast-feeding and caesarean section delivery) on breast-feeding duration in Peru using structural equation models.

Design and setting: Structural equation models were analysed with LISREL using data from the 1991-92 Peruvian Demographic and Health Survey.

Subjects: Models were tested among 6020 women whose last child was born within 5 years of the survey and among 2711 women whose last child was born 2-5 years preceding the survey.

Results: Unplanned pregnancy and socioeconomic status had a negative influence on breast-feeding duration. Prenatal care was positively associated with the timing of breast-feeding initiation in both samples and with breast-feeding duration in the whole sample. The timing of breast-feeding initiation was inversely associated with breast-feeding duration only in the sample of older children.

Conclusions: These results imply that an unplanned pregnancy, a delayed breastfeeding initiation, and higher socioeconomic status are risk factors for an earlier discontinuation of breast-feeding through complex mechanisms involving direct and indirect effects.
\end{abstract}

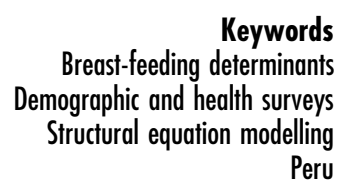

The health benefits of breast-feeding have been established in numerous studies. For instance, breastfeeding is a powerful fertility regulator ${ }^{1}$ and infants fed mother's milk experience lower morbidity and mortality rates $^{2}$. These advantages have prompted public health specialists and social scientists to devote attention to the antecedents of breast-feeding initiation and/or duration ${ }^{3-5}$. Although such efforts have uncovered a number of significant predictors, a necessary step is missing from research on breast-feeding, namely, the study of those predictors as a system or coherent model. Structural equation models have been successfully applied in the public health arena to understand how different determinants relate to each other and how they influence directly and indirectly health indicators such as child morbidity, mortality and birth weight ${ }^{6-11}$. Mosley and Chen ${ }^{6}$ were two of the earliest advocates of the use of these types of models in the health area. They proposed the analysis of a child survival model that included a set of indicators of

†Rafael Pérez-Escamilla and José A Cobas contributed equally and share first authorship of this paper. socioeconomic status, parents' values and climate as the initial variables, proximate antecedents of child mortality (such as parity, nutrition, injuries and illness) as intervening variables, and child mortality as the final variable. As they state, their model organizes 'seemingly disparate measures of environmental conditions, of dietary, reproductive, and health care practices; and of disease states into a coherent framework in which they are linked to one another and to child survival on the one hand and to socioeconomic factors on the other' ${ }^{6}$. As a result, conceptual inconsistencies, ambiguities and redundancies would stand out. Thus, the analysis of health interventions through models 'can more sharply define which among a number of specific factors amenable to change by health policymakers are of greatest consequence for child survival' ${ }^{\circ}$.

Structural equation modelling approaches are likely to improve our understanding of the determinants of maternal breast-feeding behaviour. It is particularly important to generate this knowledge in countries that are going through the epidemiological transition ${ }^{12-14}$ which involves not only a higher incidence of chronic diseases but also potential major changes in infant feeding behaviours as a result of 'modernization'15. 
Peru is a country of particular interest because it has been deeply immersed in a demographic and epidemiological transition. In the last three decades the level of urbanization has increased dramatically and the total fertility rate has declined from 6.0 to $3.6^{16}$. Furthermore, risk factors for chronic diseases such as obesity have become highly prevalent ${ }^{17}$ at a time when infectious diseases and child undernutrition are still major public health problems ${ }^{18}$. The socioeconomic inequities affecting Peru during this transition are illustrated by the country's emphasis on a free market economy at a time when the 1995 GNP per capita was only $\$ 2310.00$ and the infant mortality rate was 45 per thousand in 1996 down from 112 per thousand in $1980-85^{18}$.

Contrary to expectations, breast-feeding behaviour in Peru rose between the mid 1970s and mid 1980s possibly as a result of breast-feeding promotion and/or general increases in poverty levels ${ }^{19}$ and this rise in breast-feeding behaviour was still apparent in $1992^{20}$. Peru currently has one of the highest rates of breastfeeding initiation and average breast-feeding duration in Latin America ${ }^{20}$. In order to understand how to protect these levels of breast-feeding as Peru 'modernizes' and becomes more affected by the public health problems related to the epidemiological transition, it becomes paramount to identify the paths through which breast-feeding determinants affect this behaviour. Thus, the purpose of this paper is to study determinants of breast-feeding in Peru as an integrated system through structural equation models.

\section{Methods}

\section{Database}

Our analyses are based on the Demographic and Health Survey (DHS) conducted in Peru in 1991-92 ${ }^{16}$. DHS is a program funded by the United States Agency for International Development (USAID) and provides assistance to developing countries with the design and implementation of nationally representative surveys focusing on family planning, infant feeding practices, and maternal and child health and nutrition data. DHS replaced the World Fertility Surveys (WFS) as one of the most important sources of reliable infant feeding and family planning information worldwide. Whereas WFS collected data in the 1970s, DHS-I data were collected in the 1980s and DHS-II and -III data began to be collected in the early 1990s. The surveys are developed and implemented with the technical assistance of Macro International Inc. in Calverton, Maryland, USA. In all countries a core survey module is applied which includes detailed infant feeding questions applied to women with children 5 years old or younger.

Data for the Peruvian DHS-II survey began to be collected simultaneously in all 13 administrative regions of the country on 17 October 1991 and ended on 7 March 1992. The survey was implemented with on-site technical assistance from Macro International Inc. The sampling framework was developed by the Instituto Nacional de Estadística e Informática (INEI) by updating the national census framework using recent maps and enumerations which took place between February and December 1991. The sampling process involved a multistage stratified complex probabilistic survey leading to 910 clusters, 514 of which were in metropolitan areas and 396 representing the rest of the country. Of the 14470 eligible households and 17149 eligible women, responses were obtained from 15882 women of reproductive age (1549 years of age) living in 13479 households. The main reasons for non-responses at the household level were house 'abandoned' or 'not found', and only $1 \%$ of the target women who were contacted refused the interview ${ }^{16}$.

Fieldwork was conducted by 14 teams, one for each region and two for Lima. Each team was formed by four interviewers, one survey supervisor, one anthropometric supervisor and one driver. In addition there were five national supervisors who periodically travelled from the INEI to the field. Quality control procedures involved thorough training and supervision during sampling, data collection and data entry and cleaning following standard DHS procedures, software and manuals of operations ${ }^{16}$. The individual recoded raw database used in this study was sent to the University of Connecticut in rectangular format (i.e. one observation per subject) on floppy diskettes from the DHS headquarter. These data were read with a Pentium PC system using SPSS software distributed by DHS. A copy of this 'master' file was transferred to Arizona State University where the LISREL analyses presented in this article were performed.

\section{Statistical modelling}

LISREL $^{21}$ was used to identify the antecedents of breastfeeding in Peru using the theoretical model presented in Fig. 1. The first sample used was based on the last child born within the 5 years preceding the survey who had no missing data for any of the variables specified in the model $(n=6020 / 6335)$. In this model, breastfeeding duration was handled as a right-censored value for the $44 \%$ who were still breast-feeding at the time of the interview. Because of the high degree of censoring in this sample, the same model was replicated on the subsample of children aged between 2 and 5 years $(n=2711 / 2879)$. As a result, only $9 \%$ of the cases included in this subsample were censored for breast-feeding duration. Estimates were obtained through weighted least squares for the categorical variables and Tobit analysis for the censored variable ${ }^{21-23}$. 


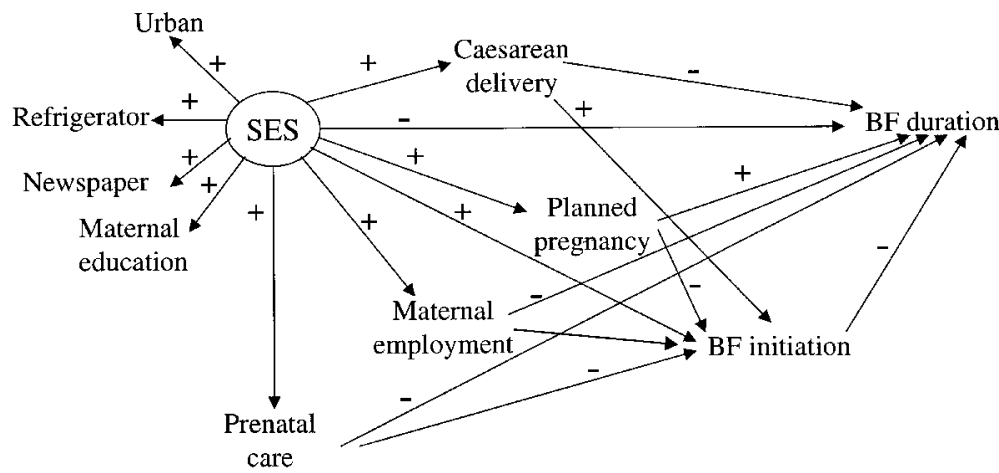

Fig. 1 Theoretical model of breast-feeding (BF) determinants in Peru

For the interpretation of results it is important to underscore that the coefficients linking socioeconomic status with its indicators, lambda coefficients in LISREL's terminology, are akin to factor loadings. The effects from the exogenous variable (socioeconomic status) to the other variables in the models are expressed as gamma coefficients, and effects from one endogenous variable to another as beta coefficients. Standardized values are provided for the lambda, beta and gamma coefficients and are useful for comparing coefficients within a model. The fit of the structural equation model was determined using two measures: critical $\mathrm{N}$ and the goodness of fit index ${ }^{22}$. The goodness of fit index taps the degree of agreement between the actual and predicted values of the covariance matrix of the variables in the model. Its maximum value is 1 . Critical $\mathrm{N}$ gives the sample size at which the hypothesis specifying that the actual and predicted covariance matrices are equal can be rejected. Other things being equal, the larger the value of $\mathrm{N}$, the easier it is to reject this hypothesis. When a model holds at substantial values of $\mathrm{N}$, there is a good fit of model and data. By convention, values of critical $\mathrm{N}$ greater than 200 indicate a good fit. According to these criteria, both of our models fit the data (Figs 2 and 3).

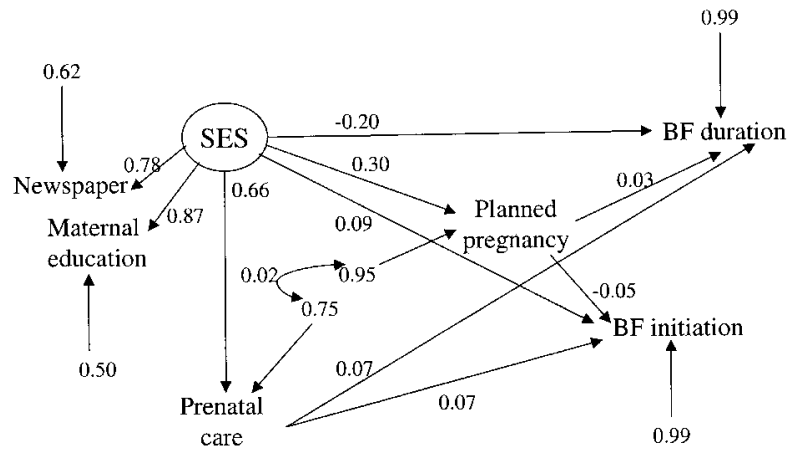

Fig. 2 LISREL Model for the entire sample $(n=6020)$. Only significant coefficients are shown. Goodness of fit index $=1.00$, critical $N=4257$

\section{Variables}

Two criteria governed the choice of the variables in the initial model. First, they had to have been shown in previous studies to be fairly consistent predictors of breast-feeding initiation and duration ${ }^{3-5}$. Second, they had to fit into a cohesive causal model. In such models, variables are classified into two categories: exogenous and endogenous. Exogenous variables are those determined outside the system; endogenous variables are determined by exogenous and/or other endogenous variables as well as by other influences not explicitly incorporated into the model (i.e. the 'error' terms).

The original theoretical model included nine manifest variables (Fig. 1). Four of these, 'urban', 'refrigerator', 'newspaper' and 'maternal education', are indicators of the model's only exogenous variable, socioeconomic status, which is also the model's only latent variable. 'Urban' was coded as 0 if the respondent lived in a rural area or 1 if residence was in an urban centre (i.e. a place with at least 2500 inhabitants). 'Refrigerator' is a dichotomous variable coded 1 if there was a refrigerator in the respondent's household, 0 if otherwise. 'Newspaper' was coded 1 if the respondent read the newspaper at least once a week, 0 if less often. 'Maternal education' ranges from 0 (no education) to 3 (completed higher than secondary education).

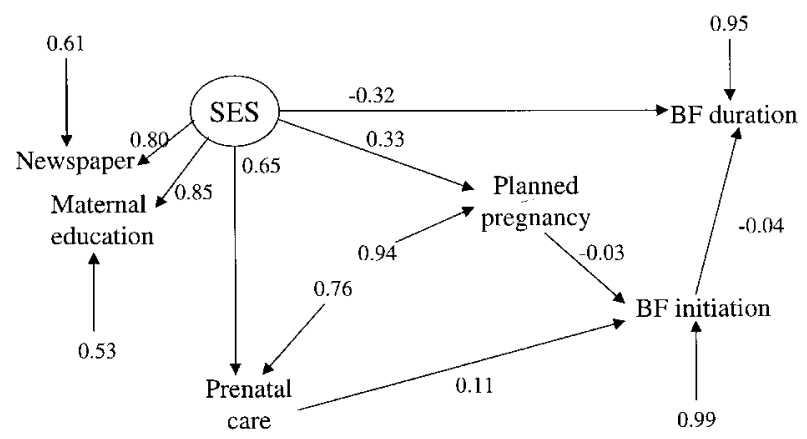

Fig. 3 LISREL Model for mothers whose last child was 24 months or older $(n=2711)$. Only significant coefficients are shown. Goodness of fit index $=1.00$, critical $N=5425$ 
'Prenatal care' was coded 1 if the respondent had one or more visits to a health facility while pregnant, 0 if otherwise. 'Maternal employment' was coded 1 if the mother worked away from the child and 0 if the mother did not work, or worked in close proximity to the child. 'Planned pregnancy' was used to indicate whether the woman was planning to have the index child when she became pregnant or if this was an unplanned event. This variable was coded 1 if the pregnancy came at a time when the mother was planning to get pregnant, 0 if otherwise. 'Caesarean section' was coded 1 if the delivery took place through this surgical procedure, 0 if otherwise. 'Breast-feeding initiation' was coded 1 if the mother began breast-feeding 24 hours after delivery or later, 0 if otherwise. The variable 'breast-feeding duration' was expressed in months and because some respondents were still breast-feeding at the time of the interview, this variable was handled as a right-censored outcome. Ninety-seven per cent of the women breastfed their children. For those who never breast-fed the index child or those who breast-fed for less than a month, a breast-feeding duration of 0.5 months was assigned.

The model posits several relationships. Socioeconomic status affects directly caesarean section, planned pregnancy, employment, prenatal care, timing of breast-feeding initiation and breast-feeding duration. Caesarean section, planned pregnancy, employment and prenatal care are predicted to have direct effects on timing of breast-feeding initiation, and all of these endogenous variables are seen as having direct effects on breast-feeding duration (Fig. 1).

Socioeconomic status has been consistently identified as a variable with a strong influence on breastfeeding outcomes. The availability of a refrigerator in the household ${ }^{24,25}$, exposure to 'modern' ideas in a newspaper and formal education ${ }^{5,19}$ are all characteristics that increase the use of mother's milk substitutes and thus decrease the probability of breast-feeding success in developing countries. Exposure to the health sector has been identified as a negative predictor of breast-feeding success ${ }^{24,26}$. Although the reasons for this link are not fully understood yet, in developing countries it is usually assumed that breast-feeding is discouraged by the formal health sector. This situation, however, has changed dramatically during recent years as a result of initiatives such as the UNICEF/WHO Baby Friendly Hospital Initiative ${ }^{25}$. Maternal attitude to breast-feeding has been consistently identified as a predictor of breast-feeding success ${ }^{3,15}$. Because crosssectional surveys usually do not collect data on breastfeeding intentions, we used the variable 'planned pregnancy' as a proxy for breast-feeding intentions. The implicit assumption was that women who got pregnant while not planning to have a child would be less motivated to start breast-feeding soon after delivery and more likely to breast-feed for shorter periods of time $^{27}$.

Since socioeconomic status is predicted to decrease the probability of breast-feeding success, it is plausible to expect that it will also delay the timing of breastfeeding initiation. Women who deliver via a caesarean section are likely to initiate breast-feeding later than their counterparts who deliver vaginally ${ }^{28}$. Higher socioeconomic status women are more likely to be employed in the formal labour sector. Maternal employment may in turn be associated with a shorter breast-feeding duration.

It further follows from the preceding discussion that mothers who were not motivated to get pregnant are more likely to delay the initiation of breast-feeding. And, as stated earlier, given recent global initiatives encouraging initiation of breast-feeding in the early postpartum period, it is reasonable to assume that those who are exposed to the health sector will also be more likely to start breast-feeding sooner. This posited relationship is also supported by the likelihood that women who do not deliver through the health sector may hold more traditional beliefs associated with the delay of breast-feeding initiation. Studies have suggested that the longer it takes for women to initiate breast-feeding, the higher the likelihood that they will introduce infant formulas and that breast-feeding will be prematurely terminated ${ }^{29}$.

Studies have established that the higher the socioeconomic status the higher the probability that a woman has access to the health sector ${ }^{24}$. Finally, the resources provided by high socioeconomic status facilitate the mother's ability to choose her mode of delivery and plan when to have children.

\section{Final model specification}

The first attempt at model fitting with the sample of children under 5 years of age produced unsatisfactory results. Part of the problem resided with 'caesarean delivery', which, due to its severe skewness (only $9 \%$ of the children were delivered through this surgical procedure), had to be dropped. 'Employment' and 'refrigerator' represented additional sources of instability, as models which included them would not fit the data. After several unsuccessful attempts to fix the problem, these two variables also had to be dropped. It is unlikely that we introduced a bias with this decision as there are other socioeconomic status variables represented in the model and previous DHS analyses have not identified maternal employment as a potent predictor of infant feeding practices ${ }^{30}$.

Even after these changes, the model-fit statistics for the analyses based on the whole sample were not adequate. Thus, the possibility that some of the error terms were correlated was examined. A correlation was found between the error terms of 'planned pregnancy' 
and 'prenatal care'. After this correlation was included, a satisfactory model fit was achieved, as shown by the fitness indices (Fig. 2). For the sample of older children the model-fit statistics were adequate without the need for inclusion of correlated errors.

\section{Results}

\section{Sample characteristics}

Almost two-third of the women interviewed resided in urban areas and the percentage residing in those areas tended to be higher in the subsample of younger children than in the subsample of older children. As expected, the urban bias was reflected with better socioeconomic indicators and more exposure to the health sector (e.g. prenatal care, delivery attended by a physician) in the subsample of older children. The demographic and epidemiological transition that Peru is undergoing is reflected in the fact that contrary to the situation a few decades ago, in 1991-92 the majority of women lived in urban areas, and were exposed to the health sector through prenatal care and the delivery of their children. A typical outcome of the epidemiological transition in Peru is the fact that in this representative sample, $41 \%$ of women were obese at a time when $26 \%$ of their children were stunted. The vast majority of women were married or lived in 'free unions', and as expected the majority were multiparous. Forty-one per cent of the women were not planning to have a child at the time when they became pregnant with the study child. Poverty is still widespread in Peru as illustrated by the fact that only one-third of the sample had a refrigerator at home. Close to half of the women did not complete secondary education and one out of every five worked away from their children. Boys and girls were evenly represented in all subsamples and breast-feeding initiation was almost universal.

\section{Breast-feeding antecedents}

\section{Sample of children under 5 years of age}

Figure 2 shows the results for the model based on the entire sample. Most of the predicted direct links are supported by this empirical model, except the link between the timing of breast-feeding initiation and breast-feeding duration.

Planned pregnancy has a positive effect, and socioeconomic status a negative effect, on breast-feeding duration. Comparison of the standardized values of the two lambda coefficients associated with socioeconomic status shows that this latent variable is influenced more by schooling than by exposure to newspapers. Prenatal care and socioeconomic status were positively associated, and planned pregnancy was inversely associated, with the timing of the initiation of breast-feeding.
Socioeconomic status has positive effects on both prenatal care and planned pregnancy. Contact with the formal health sector, measured by prenatal care, was positively associated with duration of breast-feeding.

Results from this model reveal that the relationship between breast-feeding duration and socioeconomic status is complex. First, while the direct effect of socioeconomic status on breast-feeding duration is negative, its indirect effect through prenatal care and planned pregnancy is positive. However, on balance, the overall effect of socioeconomic status on breastfeeding duration is negative as the size of its negative direct effect $(-0.20)$ is four times as large as its positive indirect effect $(0.66 \times 0.07+0.30 \times 0.03=0.05)$. Second, the magnitude of the associations resulting from the fact that socioeconomic status is a common predictor of planned pregnancy, prenatal care and breast-feeding duration is negative and larger than the direct effects on breast-feeding duration associated with these two endogenous variables. The magnitude of the association between prenatal care and breast-feeding duration due to their common dependence on socioeconomic status is equal to $-0.20 \times 0.66=-0.13$. The comparable figure for planned pregnancy is $-0.20 \times 0.30=-0.06$. Thus, while prenatal care and planned pregnancy increase breast-feeding duration, socioeconomic status works in the background to diminish much of this influence.

\section{Sample of children 2-5 years of age}

Findings for the subsample of older children (Fig. 3) were remarkably consistent with those for the subsample of all children under 5 years. An important difference is that in this model the timing of breastfeeding initiation was inversely associated with breastfeeding duration. Planned pregnancy was inversely associated and prenatal care was positively associated, with the timing of breast-feeding initiation which in turn was inversely associated with breast-feeding duration. Thus, prenatal care had a negative indirect effect on breast-feeding duration mediated by the timing of breast-feeding initiation. As expected, socioeconomic status was negatively associated with breastfeeding duration and positively with the likelihood that the child was born as a result of a planned pregnancy. Prenatal care was positively associated, and planned pregnancy was inversely associated, with the timing of the initiation of breast-feeding. Socioeconomic status has positive effects on both prenatal care and planned pregnancy.

\section{Discussion}

A substantial body of evidence exists to causally link breast-feeding with numerous positive health outcomes for children ${ }^{2}$ and women $^{31}$. In order to 
maximize the exposure of infants and women to these benefits, it is necessary to improve our understanding of breast-feeding determinants. The analyses presented here, based on a sample of Peruvian women, sheds some new light on factors that are likely to affect infant feeding decisions in a developing country. These analyses clearly showed that women whose pregnancies were not planned were at risk of breast-feeding for shorter periods of time. This finding was consistent in the entire sample and the subsample of older children where only a small proportion of cases were censored. Although this relationship has previously been reported in the $\mathrm{USA}^{27}$, to our knowledge this is the first report documenting it in a developing country setting. About $40 \%$ of pregnancies occurring in developing countries happen at a time when they are not planned. Thus, these findings have important public health implications. Results may also indicate that the 'planned pregnancy' variable may be used as a proxy for breast-feeding motivation in cross-sectional surveys where it is very difficult to collect data on breast-feeding plans before or soon after delivery.

Prenatal care was associated with a more delayed initiation of breast-feeding in both samples, and was positively associated with breast-feeding duration in only one of the two samples tested. These ambivalent findings clearly call for updated longitudinal studies examining how the public health sector is currently shaping infant feeding decisions in Peru and in other parts of the world.

The association between the timing of the initiation of breast-feeding and breast-feeding duration did not reach statistical significance in the whole sample of children under 5 but was inversely associated with breast-feeding duration in the subsample of older children. The latter is consistent with previous analyses in Latin America ${ }^{29}$ that have found such association.

As expected, socioeconomic status emerged as a powerful predictor of breast-feeding duration. This relationship, however, is complex as it involved direct and indirect effects running in opposite directions, but overall a higher socioeconomic status tends to decrease duration. The large direct effect of socioeconomic status on breast-feeding duration indicates that further work is needed to identify other factors that mediate the relationship between socioeconomic status and infant feeding behaviours.

\section{Conclusions}

The findings presented are of value for public health. First, we report in a developing country setting that unplanned pregnancies are a risk factor for shorter breast-feeding durations. Indeed, this finding has major public health implications for breast-feeding promotion efforts in Peru. Second, our analyses show that the relationships among breast-feeding determinants and between each determinant and breast-feeding duration is complex, involving direct and indirect effects that were disentangled, at least in part, thanks to the application of a structural equation model. The epidemiological transition in developing countries has usually been presented in terms of how chronic diseases are becoming a health burden at a time when these societies are still facing serious challenges related to infectious diseases. Infant feeding practices are also strongly affected by this transition because as these countries become more urbanized and women become better educated the tendency could be for a decline in the incidence and duration of breastfeeding ${ }^{32}$. Because breast milk and the process of breast-feeding offer important public health benefits to women and children ${ }^{33}$, it is important that infant feeding behaviours become part of the ongoing debate on epidemiological transition ${ }^{12-14}$.

\section{References}

1 Labbok $\mathrm{MH}$, Laukaran VH. Breast feeding and family planning. In: Dilts PV, Sciarra JJ, eds. Gynecology and Obstetrics, Vol. 6. Philadelphia: Lippincott, 1994: 1-13.

2 Heinig MJ, Dewey KG. Health advantages of breast-feeding for infants: a critical review. Nutr. Res. Rev. 1996; 9: 89-110.

3 Pérez-Escamilla R, Segura-Millán S, Pollitt E, et al. Determinants of lactation performance across time in an urban population from Mexico. Soc. Sci. Med. 1993; 37: 1069-78.

4 Popkin BM, Bilsborrow RE, Akin SA, Yamamoto ME. Breast feeding determinants in low-income countries. Med. Anthropol. 1983; 7: 1-31.

5 Forman MR. Review of research on the factors associated with choice and duration of infant feeding in less developed countries. Pediatrics 1984; 74: 667-93.

6 Mosley WH, Chen LC. An analytical framework for the study of child survival in developing countries. Popul. Dev. Rev. 1984; 10 (Suppl.): 25-45.

7 Cebu Study Team. Underlying and proximate determinants of child health: the Cebu Longitudinal Health and Nutrition Study. Am. J. Epidemiol. 1991; 133: 185-201.

8 Adelman PK, Antonucci TC, Crohan SE, Coleman LM. A causal analysis of employment and health in midlife women. Women Health 1990; 16: 5-20.

9 Taylor SA, Cronin JJ. Modeling patient satisfaction and service quality. J. Health Care Market. 1994; 14: 34-44.

10 Gruber JW, Wallack L. Television beer advertising and drinking knowledge, beliefs, and intentions among schoolchildren. Am. J. Public Health 1994; 84: 254-9.

11 Cobas JA, Balcazar H, Benin MB, et al. Acculturation and low-birthweight among Latino women: a reanalysis of HHANES data with structural equation models. Am. J. Public Health 1996; 86: 394-6.

12 Popkin BM. The nutrition transition in low income countries: an emerging crisis. Nutr. Rev. 1994; 52: 285-98.

13 Monteiro CA, Mondini L, Meeiros de Souza AL, Popkin BM. The nutrition transition in Brazil. Eur.J. Clin. Nutr. 1995; 49 $105-13$

14 World Health Organization. Investing in Health Research and Development. Geneva, Switzerland: World Health Organization, 1996.

15 Stuart-Macadam P, Dettwyler KA. Breast Feeding: Biocultural Perspectives. New York: Aldine de Gruyter, 1995. 
16 Instituto Nacional de Estadística e Informática, Asociación Benéfica PRISMA, Demographic and Health Surveys/Macro International Inc. Perú. Encuesta Demográfica y de Salud Familiar 1991/1992. Calverton, MD: Demographic and Health Surveys/Macro International Inc., 1992.

17 Martorell R, Khan LK, Hughes ML, Grummer-Strawn LM. Obesity in Latin American women and children. J. Nutr. 1998; 128: 1464-73.

18 UNICEF. The State of the World's Children. UNICEF, 1999.

19 Elo IT, Grummer-Strawn LM. Changes in breast-feeding initiation and duration in Peru, 1977-1986. Soc. Biol. 1993; 40: 224-43.

20 Labbok M, Pérez-Escamilla R, Peterson AE, Coly S. Breastfeeding and Child Spacing: Country Profiles. Washington, DC: Institute for Reproductive Health, 1997.

21 SPSS Inc. SPSS LISREL 7 and PRELIS User's Guide and Reference. Chicago: SPSS Inc., 1990.

22 Muthen BA. General structural equation model with dichotomous, ordered categorical, and continuous latent variable indicators. Psychometrika 1984; 49: 115-32.

23 Muthen B. Tobit factor analysis. Br. J. Math. Stat. Psychol. 1989; 42: 241-50.

24 Adair LS, Popkin BM, Guilkey DK. The duration of breastfeeding: how is it affected by biological, sociodemographic, health sector, and food industry factors? Demography 1993; 30: 63-80.

25 Pérez-Escamilla R, Lutter CK, Wickham C, et al. Identification of risk factors for short breast-feeding duration in Mexico City through survival analysis. Ecol. Food Nutr. 1997; 36: 43-64.
26 Mock NB, Franklin RR, Bertrand WE, et al. Exposure of the modern health service as a predictor of the duration of breast-feeding: a cross-cultural study. Med. Anthropol. 1985; Spring: $123-38$.

27 Dye TD, Wojtowycz MA, Aubry RH, Quade J, Kilburn H. Unintended pregnancy and breast-feeding behavior. Am. J. Public Health 1997; 87: 1709-11.

28 Pérez-Escamilla R, Segura-Millán S, Canahuati J, et al Prelacteal feeds are negatively associated with breastfeeding outcomes in Honduras. J. Nutr. 1996; 126: $2765-$ 73.

29 Perez-Escamilla R, Maulen-Radovan I, Dewey KG. The association between Cesarean delivery and breast-feeding outcomes among Mexican women. Am. J. Public Health 1996; 86: 832-6.

30 Hight-Laukaran V, Rutstein SO, Peterson AE, et al. The use of breastmilk substitutes in developing countries: the impact of women's employment. Am. J. Public Health 1996; 86: 123540.

31 Micozzi MS. Breast cancer, reproductive biology, and breastfeeding. In: Stuart-Macadam P, Dettwyler KA, eds. Breastfeeding: Biocultural Perspectives. New York: Aldine de Gruyter, 1995: 347-84.

32 Pérez-Escamilla R. Breast-feeding patterns in Latin America and the Caribbean. Bull. Pan Am. Health Organ. 1993; 27: $32-42$.

33 Horton S, Sanghvi T, Phillips $\mathrm{M}$, et al. Breastfeeding promotion and priority setting in health. Health Policy Plan. 1996; 11: 156-68. 